\title{
Mollusca of the Magellan region. A checklist of the species and their distribution*
}

\author{
KATRIN LINSE \\ Zoological Institute and Zoological Museum, University of Hamburg, Martin-Luther-King-Platz 3, \\ D-20146 Hamburg, Germany.
}

\begin{abstract}
SUMMARY: The Molluscan species collected during the "Victor Hensen" Joint Magellan Campaign 1994 served as the basis for this species list. The list was then completed by literature data on the distribution of molluscs around the Falkland Islands, the other Scotia Arc Islands, Antarctica and the Kerguelen Islands. 397 Magellan species are known: 10 species of Aplacophora, 250 species of Gastropoda, 6 species of Scaphopoda and 131 species of Bivalvia. Polyplacophora and Cephalopoda are not included because both taxa need revision.
\end{abstract}

Key words: Mollusca, Magellan region, checklist, biogeography.

RESUMEN: Moluscos De LA REGión DE MAgallanes. Lista DE ESPECIES Y SU DISTRIBUCIÓN. - La base para esta lista está formada por las especies de moluscos recolectados durante la campaña internacional multidisciplinaria magallánica "Victor Hensen" en el año 1994. La lista se completó con datos de la literatura y estos se compararon con los datos existentes sobre la distribución en las Islas Malvinas, las Islas del Arco de Escocia, Antártida y las Islas Kerguelen. La lista de moluscos de Magallanes se compone de 397 especies: 10 especies de aplacóforos, 250 especies de gasterópodos, 6 especies de escafópodos y 131 especies de bivalvos. Polyplacóforos y cefalópodos no están incorporados en esta lista porque ambos taxones necesitan revisión.

Palabras clave: Moluscos, región de Magallanes, lista de especies, biogeografía.

\section{INTRODUCTION}

This species list will serve to provide an inventory of the marine Magellanic Mollusca. Since the last century many expeditions have been undertaken to the Magellan region. The first taxonomic studies on molluscs were performed by King and Broderip (1831), D’Orbigny (1835-1846) and Philippi (1845). Later, important results on the Magellanic malacofauna were published by Smith (1881), Mabille and Rochebrune (1889), Strebel (1904, 1095a, b, 1906, 1907, 1908) and Odhner (1926). After expeditions with RVs Challenger, Belgica,

\footnotetext{
*Accepted September 17, 1998.
}

Albatross and Discovery (Bergh, 1884; Smith, 1885; Pelseneer, 1903; Dall, 1890, 1908; Powell, 1951; Dell,1964), the Scottish National Antarctic Expedition (Melvill and Standen, 1907, 1912), and the Swedish Lund University Chile Expedition (Marcus, 1959; Soot-Ryen, 1959) further taxonomic results were published.

Carcelles and Williamson (1951) published the first species list of marine molluscs from the Magellan Province and later Osorio and Bahamonde (1970) gave a preliminary list of Chilean bivalves, comprising 217 species with 97 of them recorded in the Magellan region. Since those published accounts taxonomic revisions (e.g. McLean, 1984; Castellanos, 1989-1993; Ponder and Worsfold, 1994; 
Schrödl, 1996) have been made for several taxa. Therefore Carcelles and Williamson's lists had to be checked for synonyms and new species. Regional zoogeographic studies of the Chilean marine benthos including Polyplacophora, Gastropoda and Bivalvia were performed by Brattström and Johanssen (1983). However, these authors mainly examined shallow water taxa; the sublittoral fauna was under-represented in their account.

In recent years new expeditions were undertaken to the Magellan region, like the Chilean-Italian cruises in 1989-90 and 1990-91 or the GermanChilean-Italian ,Joint Magellan“ campaign in 1994. Some new species were discovered during taxonomic studies of these new molluscan samples (e.g. Di Geronimo et al., 1995). Linse (1997) published an inventory of marine Mollusca of the Beagle Channel. The biogeography of Crustacea and Mollusca is presented and discussed by Brandt et al. (this volume), zoogeographic relationships of the Nudibranchia by Schrödl (1997; 1999).

The present list includes also the Aplacophora (Caudofoveata and Solenogastres), Gastropoda, Scaphopoda and Bivalvia. In total 118 molluscan species were collected in the Beagle Channel by means of an epibenthic sledge (Linse, 1997; Linse and Brandt, in press), these species served as the basis for this checklist. It was later completed with distribution data from the literature from the areas of the Subantarctic Islands and High Antarctica (e.g., Powell, 1960; Mühlenhardt-Siegel, 1989; Hain, 1990).

The Magellan region in this study is defined as the Patagonian shelf south of about $41^{\circ} \mathrm{S}$ on both the Pacific and Atlantic sides. Although Powell (1965) excluded the Falkland Islands from the Magellan region, they are included in this study (compare Brandt et al., this volume).

\section{SPECIES CHECKLIST}

(Geographic abbreviations at the end of the list.)

\section{Aplacophora \\ Caudofoveata}

\section{Limifossoridae}

Limifossor holopeltatus Salvini Plawen, 1986 RC, MR

Scutopus chilensis Salvini-Plawen, 1972 RC, MR

Scutopus sp.

MR

Chaetodermatidae

Chaetoderma sp.

Falcidens sp.1
Falcidens sp. 2

MR

Caudofoveata sp.

MR

\section{Solenogastres}

Pararrhopaliidae sp.

MR

Macellomeniidae sp.

MR

Dondersiidae sp.

MR

\section{Patellidae}

\section{Gastropoda}

Nacella (Patinigera) deaurata deaurata (Gmelin, 1791)

MR, FI

Nacella (P.) deaurata f. delicatissima (Strebel, 1907) MR, FI

Nacella (P.) flammea (Gmelin, 1791)

MR

Nacella (P.) fugiensis (Reeve, 1855)

MR, FI

Nacella (P.) magellanica magellanica

(Gmelin, 1791)

$\mathrm{RC}, \mathrm{MR}, \mathrm{FI}$

Nacella (P.) magellanica venosa (Reeve, 1855)MR

Nacella (P.) mytilina (Helbing, 1779) MR, FI, KI

\section{Acmaeidae}

Acmaea perconica Preston, 1913 PE, RC, MR

Patelloida ceciliana magellanica Strebel, 1907MR, FI

Scurria scurra (Lesson, 1847)

$\mathrm{MR}, \mathrm{FI}$

\section{Lepetidae}

Iothia coppingeri (Smith, 1881) MR, FI, SS, SO,

Fissurellidae

PI, WS, EL, DS, AD, OL, RS, PA, CI, KI

Parmaphoridea antarctica (Strebel, 1907) MR

Parmaphoridea melvilli (Thiele, 1912) MR, FI, BB

Puncturella cognata (Gould, 1852) MR

Puncturella conica (Orbigny, 1841) MR, FI, SG,

SO, WS, RS, KI

Megatebennus patagonicus Strebel, 1907 MR, FI

Fissurella nigra Lesson, 1831

Fissurella picta (Gmelin, 1791)

Fissurella oriens Sowerby, 1835

$\mathrm{RC}, \mathrm{MR}$

Fissurella radiosa Lesson, 1831

$\mathrm{RC}, \mathrm{MR}, \mathrm{FI}$

MR, FI

Scissurellidae

Anatoma conica (Orbigny, 1841) MR, FI

Scissurella clathrata Strebel, $1908 \quad$ MR, FI

Scissurella timora Melvill \& Standen, 1912 MR, FI, SO

\section{Trochidae}

Calliostoma consimilis (Smith, 1881) MR

Calliostoma coppingeri (Smith, 1880) MR

Calliostoma irisans Strebel, $1905 \quad$ MR, FI

Calliostoma mobiusi Strebel, 1905 MR, BB

Calliostoma modulestum Strebel, 1908 MR, FI

Calliostoma nordenskjoldi Strebel, 1908 MR

Calliostoma nudisculum (Martens, 1881) MR

Calliostoma nudum (Philippi, 1895) MR, FI

Calliostoma sp.

MR 
Margarella pruinosa (Mabille \& Rochebrune, 1891) MR Margarella violacea (King \& Broderip, 1831) MR, FI Photinastoma taeniata (Wood, 1825) MR, FI Photinula blakei (Clench \& Aguayo, 1938) MR, FI Photinula caerulescens (King \& Broderip, 1831)

MR, FI, SG

Tegula atra (Lesson, 1839) PE, RC, MR, FI Tegula patagonica (Orbigny, 1835) RA, MR Turbinidae

Homalopoma cunninghami (Smith, 1881) MR, FI Prisogaster niger (Wood, 1828) PE, RC, MR Cyclostrematidae

Cyclostrema crassiolatum Strebel, 1908 MR, SG Orbitestellidae

Microdiscula sp.

Orbitestella sp.

MR

Cerithiidae

Ataxocerithium pullum (Philippi, 1845)MR, FI, BB

Cerithiopsilla burdwoodianus (Melvill \& Standen, 1912)

MR, FI

Eumetula michaelseni (Strebel, 1906)

MR

Turritellidae

Colpospirella algida (Melvill \& Standen, 1912)

MR, FI, SG

Mathilda argentina Castellanos, $1990 \quad$ MR

Mathilda malvinarum (Melvill \& Standen, 1907)

\section{Epitoniidae}

MR, FI

Coroniscala magellanica (Philippi, 1845) MR, FI, BB

Coroniscala latecostata (Strebel, 1905) MR, FI

Epitonium georgiettinum (Kiener, 1839) MR, RA

Epitonium fabrizioi Pastorino \& Penchaszadeh, 1999 MR

Papuliscala diminuta Castellanos, Rolan, Bartolotta, 1987

\section{Eulimidae}

MR

Balcis subantarcticus (Strebel, 1908) MR, SG Melanella salvadori Castellanos, Rolan, Bartolotta, 1987

MR

Strombiformis carforti (Rochebrune \& Mabille, 1889)MR Littorinidae

Laevilitorina calignosa (Gould, 1848) MR, FI, SG, SS, SO, KI, MA

\section{Eatoniellidae}

Eatoniella (Eatoniella) cana Ponder, 1983 MR, FI, WS, SG, SO

Eatoniella (E.) castanea Ponder, 1994 MR

Eatoniella (E.) denticulata Ponder, 1994 MR

Eatoniella (E.) glomerosa Ponder, $1994 \quad$ MR

Eatoniella (E.) nigra (Orbigny, 1840) RC, MR

Eatoniella (E.) picea Ponder, 1994

Eatoniella (E.) turricula Ponder, 1994

Pupatonia magellanica Ponder, 1990

\section{Cingulopsidae}

Skenella (Skenella) hallae Ponder, 1994MR, FI, SG

Rissoidae

Onoba ? algida Ponder, $1994 \quad$ MR

Onoba erugata Ponder, 1994 MR

Onoba fuegoensis (Stebel, 1908) MR

Onoba georgiana (Pfeffer, 1886) MR, FI, SG, SO

Onoba? lacuniformis Ponder, $1994 \quad$ MR, FI

Onoba protofimbriata Ponder, $1994 \quad$ MR, FI

Onoba scythei (Philippi, 1868) MR, FI

Onoba striola Ponder, $1994 \quad$ MR

Onoba subaedonis Ponder, $1994 \quad$ MR, FI

Onoba subincisa Ponder, 1994 MR

Onoba sulculata Ponder, 1994 MR

Powellisetia microlirata Ponder, 1994 RC, MR

Pusillina (Haurakia) averni Ponder, 1994 MR

Caecidae

Fartulum magellanicum Di Geronimo, Privitera \& Val-

dovinos, 1995

MR

Vermetidae

Vermetidae sp.

Calyptraeidae

Crepipatella dilatata Lamarck, $1822 \quad$ MR, FI

Trochita pileus (Lamarck, 1822) MR, FI, BB, RC

Trochita pileolus (Orbigny, 1845) MR, FI, BB

Capulidae

Capulus chilensis Dall, $1904 \quad$ MR

Capulus compressus Smith, $1891 \quad$ MR

Lamellariidae

Marseniopsis pacifica Bergh, 1886 MR, SG, SO, PI, WS, CI, KI

Lamellaria ampla Strebel, 1906

MR, FI

Lamellaria elata Strebel, 1906

MR, FI

Lamellaria patagonica Smith, 1881

MR, FI

\section{Cymatiidae}

Argobuccinum argus (Gmelin, 1790) MR

Argobuccinum cancellatum (Lamarck, 1822) MR, FI

\section{Naticidae}

Bulbus carcellesi Dell, 1990

MR, FI

Falsilunatia limbata (Orbigny, 1835) MR, RA

Falsilunatia patagonica (Philippi, 1845) MR, FI, SG

Falsilunatia soluta (Gould, 1848) MR, FI, CI, KI

Tectonatica impervia (Philippi, 1845) MR, FI, BB, SG

Turridae

Aforia gonoides (Watson, 1881) MR, FI, SH

Belalora cunninghami (Smith, 1881) MR, FI

Drillia janseni Strebel, 1905 MR

Drillia patagonia (Orbigny, 1841) MR

Drillia suxdorfi Strebel, $1905 \quad$ MR

Eumetadrillia fuegiensis (Smith, 1888)MR, RA, FI

Leucosyrinx angusteplicata (Strebel, 1905)MR, FI, BB

Leucosyrinx taludana Castellanos \& Landoni, 1993 MR 
Mangelia gazellae (Strebel, 1905)

Mangelia magellanica (Martens, 1881)

Mangelia martensi (Strebel, 1905)

MR,

MR

MR

Mangelia michaelseni (Strebel, 1905) MR, FI, BB,

SG

Mangelia paessleri (Strebel, 1905)

MR

Pleurotomella (Anomalotomella) ohlini (Strebel, 1905)

MR, FI

Propebela profunda Castellanos \& Landoni, 1993

MR

Propebela? lateplicata Strebel, 1905

MR

Thesbia michaelseni Strebel, 1905

Typhlodaphne filostriata (Strebel, 1905)

MR

MR

Typhlodaphne payeni (Rochebrune \& Mabille, 1891) MR

Typhlodaphne purissima (Strebel, 1908) MR, SR, SG

Typhlodaphne strebeli Powell, 1951 MR, SR, SG

Columbellidae

Amphissa acuminata (Smith, 1915) MR, RA, FI

Amphissa cancellata (Castellanos, 1979) MR,

Anachis isabellei (Orbigny, 1841) MR, RA

Anachis paessleri (Strebel, 1905) MR, RA

Mitrella unifasciata (Sowerby, 1832) RC, MR, RA

Mitrella rubra (Martens, 1881) MR, RA

Buccinulidae

Anomacme smithi Strebel, 1908

MR, FI

Antistreptus magellanicus Dall, 1902

Antistreptus rolani Castellanos, 1985

Parabuccinum* bisculpta (Dell, 1990)

Parabuccinum* eltanini (Dell, 1990)

MR, FI

MR

MR

MR

Parabuccinum* polyspeira (Dell, 1990)

MR

Parabuccinum* rauscherti sp. n. Harasewych,

Kantor \& Linse, (subm.)

Falsitromina bella (Powell, 1951)

Falsitromina fenestrata (Powell, 1951)

Falsitromina powelli Dell, 1990

$\mathrm{MR}, \mathrm{FI}$

MR

MR, FI

Falsitromina simplex (Powell, 1951) MR, FI, BB

Glypteuthria meridionalis (Smith, 1881) MR, FI

Meteuthria martensi (Strebel, 1905)

MR

Meteuthria multituberculata multituberculata

(Castellanos, Rolan \& Bartolotta, 1987)

Parficulina problematica Powell, 195

MR

Pareuthria cerealis (Rochebrune \& Mabille, 1885)MR, FI

Pareuthria fuscata (Bruguière, 1782) MR, FI

Pareuthria janseni (Strebel, 1905)

Pareuthria michaelseni (Strebel, 1905)

Pareuthria paessleri (Strebel, 1905)

Pareuthria plumbea (Philippi, 1884)

Pareuthria ringei (Strebel, 1905)

MR, FI

MR, FI

MR

MR

Pareuthria powelli (Hombron \& Jacquinot, 1854)

MR, FI, BB
Pareuthria valdiviae Powell, 1951

MR

Prosipho antarctidis (Pelseneer, 1903) MR, BB

Savatieria areolata Strebel, 1905 MR, FI, BB

Savatieria chordata Castellanos, Rolan \& Bartolotta, 1987

MR

Savatieria frigida Rochebrune \& Mabille, 1885MR

Savatieria meridionalis (Smith, 1881) MR

\section{Volutidae}

Adelomelon ancilla (Solander, 1786) MR, RA, FI

Adelomelon beckii (Broderip, 1836) MR, RA, FI

Adelomelon ferussacii (Donovan, 1824) MR

Miomelon? bottai Vazquez \& Caldini, 1989 MR

Miomelon eltanini Dell, 1990

MR

Miomelon scoresbyana Powell, $1951 \quad$ MR, FI

Miomelon turnerae Dell, $1990 \quad$ MR

Odontocymbiola canigiai Vasquez \& Caldini, 1992 MR

Odontocymbiola magellanica (Gmelin, 1791) MR, FI

Odontocymbiola pescalia Clench \& Turner, 1964 MR

Odontocymbiola subnodosa Leach, 1914 MR, FI

Volutomitridae

Volutomitra (Paradmete) crymochara (Rochebrune

\& Mabille, 1889)

MR

Cancellariidae

Admete cf. australis (Philippi, 1856)

MR

Admete carinata (Watson, 1883)

MR, FI, KI

Admete magellanica Strebel, 1905

MR, FI

Marginellidae

Marginella dozei Rochebrune \& Mabille, 1889 MR, FI

Marginella warrenii Marrat, 1876

MR, FI

Muricidae

Tromina dispectata Dell, 1990

MR

Trophon geversianus (Pallas, 1769)

MR, FI

Trophon ohlini Strebel, 1905

MR, FI, SG

Trophon triacanthus Castellanos, Rolan \& Bartolotta, 1987

MR

Trophon (Fuegotrophon) pallidus (Broderip, 1832)

MR, FI, BB

Trophon (Stramonitrophon) plicatus (Lightfoot, 1786)

MR, RA, FI, BB, SG

Xymenopsis albidus (Philippi, 1846) MR, FI

Xymenopsis falklandicus (Philippi, 1845) MR, FI

Xymenopsis muriciformis (King \& Broderip, 1832)

MR, FI, SG

Acanthina monodon (Solander, 1786) MR, FI

Concholepas concholepas (Bruguiere, 1789) PE,

RC, MR

Pyramidellidae

Chemnitzia smithi (Strebel, 1905) MR, FI, BB

Eulimella xenophyses (Melvill \& Standen, 1912)

MR, FI, BB

Eulimella sp.

MR

Odostomia translucens Strebel, 1908

MR, FI 
Odostomia multituberculata Castellanos, 1982 MR

Odostomia nova Castellanos, $1982 \quad$ MR

Omalogyridae

Omalogyra antarctica Egorova, 1991 MR, AD

Acteonidae

Acteon biplicatus (Strebel, 1908) MR, FI

Acteon elongatus Castellanos, Rolan \& Bartolotta, 1987

MR

Acteon vagabundus (Mabille \& Rochebrune, 1885)

MR

Diaphanidae

Diaphana paessleri (Strebel, 1905) MR, FI

Toledonia limnaeformis (Smith, 1877) MR, RS, KI

Toledonia parelata Dell, 1990

Toledonia perplexa Dall, 1902

MR

MR,FI

Toledonia punctata Thiele, 1912 MR, SG, RS, KI

Toledonia sp.

MR

Scaphandridae

Cylichnina cumberlandiana Strebel, 1908 MR, SG

Cylichnina georgiana Strebel, $1908 \quad$ MR, SG

Scaphandridae sp.

MR

Philinidae

Philine falklandica Powell, $1951 \quad$ MR, FI

Limacinidae

Limacina helicina (Phipps, 1774)MR, cosmopolitic

Limacina retroversa (Fleming, 1823) MR, RA

Thilea procera Strebel, 1908

Pneumodermatidae

MR

Spongiobranchaea australis (d'Orbigny, 1836)MR,

\section{Clionidae}

SG, SS, RS, south of NZL

Clione antarctica (Smith, 1902) MR, SG, SS, AD,

$\mathrm{RS}, \mathrm{BI}$

\section{Elysiidae}

Elysia patagonica Muniaín \& Ortea, 1997

MR

Stiligeridae

Ercolania evelinae (Marcus, 1959)

$\mathrm{RC}, \mathrm{MR}$

Hermaeidae

Aplysiopsis brattströmi (Marcus, 1959) RC, MR

Limapontiidae

Limapontia sp.

Pleurobranchidae

Berthella patagonica (d'Orbigny, 1837) RC, MR

Berthella platei (Bergh, 1898) MR

Chromodorididae

Cadlina sparsa (Odhner, 1921)

fornien

Tyrinna nobilis Bergh, 1898

Rostangidae

Rostanga pulchra MacFarland, 1905

Platydorididae

RC, MR, CaliCalifornien
Gargamella immaculata Bergh, 1894RC, MR, RA,

Discodorididae

Anisodoris fontaini (d'Orbigny, 1837)RC, MR, RA

Anisodoris punctuolata (d'Orbigny, 1837) RC, MR

Neodoris claurina Marcus, 1959 MR

Diaulula hispida (d'Orbigny, 1937) RC, MR

Diaulula vestita (Abraham, 1877) MR, RA, FI

Geitodoris patagonica Odhner, 1926 MR, FI

\section{Dorididae}

Austrodoris kerguelenensis (Bergh, 1884) MR, FI,

Corambidae

$\mathrm{SH}, \mathrm{SG}, \mathrm{SO}, \mathrm{SH}, \mathrm{WS}, \mathrm{RS}, \mathrm{MI}, \mathrm{KI}, \mathrm{HI}$

Corambe lucea Marcus, $1959 \quad$ RC, MR

Onchidorididae

Acanthodoris falklandica Eliot, 1907 RC, MR, FI

Goniodorididae

Ancula fuegiensis Odhner, $1926 \quad$ RC, MR

Okenia angelensis Lance, 1966 RC, MR,

California to Baja California

Polyceridae

Polycera priva Marcus, 1959 MR

Polycera marplatensis Franceschi, 1928 MR, RA

Holoplocamus papposus Odhner, 1926 RC, MR

Thecacera darwini Pruvot-Fol, 1950 RC, MR

Dotoidae

Doto uva Marcus, $1955 \quad$ RC, MR, Brazil

Tritoniidae

Tritonia australis (Bergh, 1898) RC, MR, RA

Tritonia challengeriana Bergh, 1884 MR, FI, SG, SO, SH, WS, AD, RS

Tritonia odhneri Marcus, $1959 \quad$ RC, MR

Tritonia vorax (Odhner, 1926) MR, BB, SG

Flabellinidae

Flabellina falklandica (Eliot, 1907) MR, FI, SR, SG, CI

Eubranchidae

Eubranchus agrius Marcus, $1959 \quad$ MR

Eubranchus fuegiensis Odhner, $1926 \quad$ MR

Tergipedidae

MR Cuthona georgiana (Peffer in Martens \& Pfeffer, 1886)

MR, RS, KI

Cuthona odhneri Marcus, 1959 MR

Cuthona sp. RC, MR

Cuthona valentini (Eliot, 1907) MR, FI

Facelinidae

Phidiana lottini (Lesson, 1831) PE, RC, MR

RC, MR Phidiana patagonica (d'Orbigny, 1837) MR, RA

Aeolidiidae

RC, MR, Aeolidia papillosa var. serotina (Bergh, 1873) RC,

\section{Siphonariidae}

MR, FI 
Kerguelenella lateralis (Gould, 1846) MR, FI, SG, $\mathrm{KI}, \mathrm{CA}, \mathrm{AU}$

Pachysiphonaria lessoni (Blainville, 1824)PE, RC,

$\mathrm{MR}, \mathrm{KI}$

\section{Dentaliidae}

\section{Scaphopoda}

Dentalium lebruni Mabille \& Rochebrune, 1889 MR

Dentalium megathyris Dall, 1889 EC, PE, RC, MR

Fissidentalium majorinum (Mabille \& Rochebrune, 1889) MR, FI, SH, PI, WS, EL, DS, AD, RS

Rhabdus perceptum (Mabille \& Rochebrune, 1889) MR

Siphonodentaliidae

Cadulus dalli dalli Pilsbry \& Sharp, $1898 \quad$ MR

Pusellum falklandicum (Dell, 1964)

\section{Nuculidae}

\section{Bivalvia}

Nucula exigua Sowerby, 1832

Nucula falklandica Preston, 1912

Nucula sp.1

Ennucula eltanini Dell, 1990

Ennucula grayi (Orbigny, 1846)

Ennucula puelcha (Orbigny, 1846)

$\mathrm{RC}, \mathrm{MR}, \mathrm{RA}$

MR

MR

MR

MR

MR, FI

Linucula pisum (Sowerby, 1832) RC, MR, RA, FI

Nuculanidae

Yoldia (Aequiyoldia) eightsii (Couthouy, 1839)MR, FI, SG, SO, SH, PI, WS, RS, MB, BS, PA, KI

Yoldia (A.) woodwardi (Hanley, 1860) MR, FI, SG, $\mathrm{SO}, \mathrm{SH}, \mathrm{BS}, \mathrm{PA}$

Yoldiella chilenica Dall, 1908

Yoldiella granula Dall, 1908

MR

Yoldiella indolens Dall, 1908

MR

MR

Yoldiella infrequenz. Dall, 1908

MR

Yoldiella valettei (Lamy, 1906)

MR

Malletiidae

Malletia cumingi (Hanley, 1860)

MR, RA, FI

Malletia inequalis Dall, 1908

Malletia magellanica Smith, 1881

MR

Tindariopsis sulcata (Couthouy, 1852) MR, RA, FI

Tindaria virens Dall, 1889

MR, RA

Siliculidae

Propeleda longicaudata (Thiele, 1912) MR, SG,

SO, SH, PI, WS, EL, DS, AD, OL, RS, MB, BS

Silicula patagonica Dall, 1908

Solemyidae

Solemya macrodactyla (Mabille \& Rochebrune,1891)

MR, RA

Solemya sp. 1

MR

Limopsidae

Limopsis hirtella Rochebrune \& Mabille, 1889MR,

Limopsis knudseni Dell, 1990

FI, BS, PA

MR, SG

Limopsis marionensis Smith, 1885 MR, SG, SS,

SO, SH, PI, WS, EL, DS, AD,

OL, RS, MB,BS, PA, MI, KI

Limopsis tenella dalli Lamy, 1912EC, PE, RC, MR

Philobryidae

Philobrya sublaevis Pelseneer, 1903 MR, RA, FI, SR, SG, SH, EL, DS, AD, OL, PA, BI

Philobrya wandelensis Lamy, 1906 MR, BB, SG,

SS, SO, SH, PI, EL, DS, AD, OL, RS

Philobrya quadrata

MR, FI, SG, MI

Philobrya sp. 1

MR

Lissarca miliaris (Philippi, 1845)MR, SG, SO, SH,

Mytilidae

PI, PA, KI

Aulacomya ater ater (Molina, 1782) PE, RC, MR,

FI, KI

Mytilus edulis chilensis Lamarck, 1819 MR, FI

Choromytilus chorus (Molina, 1782) PE, RC,

MR, FI

Perna perna (Linnaeus, 1758) RC, MR, FI

Dacrydium panamensis Knudsen, $1970 \quad$ MR

Crenella sp.

MR

Pectinidae

Chlamys natans (Philippi, 1845) MR

Chlamys patagonica (King \& Broderip, 1831) MR, FI, PA

Palliolum vitreum (King, 1831)

MR, FI

Pecten pycnolepis Martens, 1881

MR

Camponectus subhyalinus (Smith, 1886) MR

Cyclopecten falklandicus Dell, 1964 MR

Cyclopecten sp. 1

MR

Cyclopecten sp. 2

MR

Limidae

Acesta patagonica (Dall, 1904)

RC, MR

Limatula hodgsoni (Smith, 1907) MR, SR, SG,

SO, SH, WS, EL, AD, RS, PA, BI

Limatula pygmaea (Philippi,1845) MR, FI, SG, SS,

Limatula sp. 1

SO, WS, BS, PA, BI, KI, MA

Thyasiridae

Genaxinus debilis (Thiele, 1912) MR, FI, SR, SG, SS, SO, SH, PI, EL, DS, RS, MB

Thyasira falklandica (Smith, 1885) (MR), FI, SO,

$\mathrm{SH}, \mathrm{PA}$

Thyasira cf falklandica (Smith, 1885) MR

Lucinidae

Loripes pertenuis Smith, 1881

MR

Lucinoma lamellata (Smith, 1881)

Kelliidae

Kellia bullata Philippi, 1845

Kellia magellanica Smith, 1881

Kellia simulans Smith, 1907
MR

MR, FI

MR, FI, KI

SH, RS 
Kellia tumbesiana (Stempell, 1899)

Erycinidae

Aligena pisum Dall, 1908

Lasaea miliaris (Philippi, 1845)

Lasaea petitiana Recluz, 1843

Lasaea sp.1

Leptonidae

Solecardia antarctica Hedley, 1911 MR, SH, RS

Solecardia sp.

\section{Montacutidae}

Mysella mabillei (Dall, 1908) MR, SR, SG, SO,

SH, PI, WS, BS, PA, MI, KI

Mysella miniuscula (Martens \& Pfeffer, 1908) MR

Mysella sculpta Soot-Ryen, 1957

MR

Cyamiidae

Cyamium antarcticum Philippi, $1845 \quad$ MR, FI

Cyamium falklandicum Melville \& Standen, 1898

MR, FI

Cyamiocardium denticulatum (Smith, 1907) MR,

FI, BB, SH, WS, EL, DS, AD, OL, RS, BS, PA, BI, KI

Cyamiomactra sp.1

MR

Cyamiomactra sp.2

Cyamiomactra sp. 3

MR

MR

Neoleptonidae

Neolepton falklandicum Dell, 1964

Neolepton hupei Soot-Ryen, 1957

Neolepton umbonatum Smith, 1885

Gaimardiidae

Gaimardia magellanica (Rousseau, 1853) MR

Gaimardia trapesina trapesina (Lamarck, 1819)

MR, FI, SG, SS, CI, KI

Kidderia exilis (H. \& A. Adams, 1863) MR, KI

Kidderia pulsilla (Gould, 1850) MR, MA

Carditidae

Carditella naviformis (Reeve, 1843)

Carditella pallida Smith, 1881

MR, FI

$\mathrm{MR}, \mathrm{BB}$

MR

Carditella pygmaea (Philippi, 1860)

Carditella tegulata (Reeve, 1843) PE, RC, MR

Carditopsis flabellum flabellum (Reeve, 1843) MR

Carditopsis semen (Reeve, 1843)

MR

Cyclocardia astartoides (Martens, 1878) MR, SG,

SS, SO, SH, PI, WS, DS,

AD, RS, BS, PA, BI, KI, AU

Cyclocardia compressa (Reeve, 1843)

MR

Cyclocardia paetelina (Clessin, 1888)

Cyclocardia spurca (Sowerby, 1832)

Cyclocardia thouarsi (Orbigny, 1846)

Cyclocardia velutina Smith, 1881

Astartidae

Astarte longirostris Orbigny, 1846

MR

MR

MR, FI

MR, FI

MR, FI, BB,

\section{Cardiidae}

SG, SH, WS, DS, MI, KI

Trachycardium delicatulum (Smith, 1915) MR, FI Mactridae

Mulinia byronensis (Gray, 1838) PE, RC, MR, FI

Mulinia edulis (King, 1831) PE, RC, MR

Mulinia laevicardo (Smith, 1881) MR

Darina solenoides King, $1831 \quad$ MR, FI

Solenidae

Ensis macha (Molina, 1782) $\quad$ RC, MR

Veneridae

Ameghinomya antiqua (King, 1831) PE, RC, MR

Gomphina foveolata (Cooper \& Preston, 1910) MR, FI

Eurhomalea exalbida (Chemnitz, 1795) MR, FI

Eurhomalea lenticularis (Sowerby, 1835) MR

Pitar rostrata (Koch, 1844)

Tawera gayi (Hupe, 1854)

MR

Tellinidae

Macoma inornata (Hanley, 1844) MR, FI

Myidae

Sphenia hatcheri Pilsbry, 1899

MR

Hiatellidae

Hiatella solida (Sowerby, 1834) MR, FI, BB, SG,

\section{Pholadidae}

MI, KI, MA

Barnea subtruncata lamellosa (Orbigny, 1846)EC, MR

Teredinidae

Bankia martensi Stempell, $1899 \quad$ MR

Pandoridae

Pandora braziliensis (Sowerby, 1874) MR

Pandora cistula Gould, $1850 \quad$ MR

Pandora patagonica Dall,1915 MR

Thraciidae

Thracia meridionalis Smith, 1885 MR, FI, SR, SG, SS, SO, SH, PI, WS, DS, RS, PA, MI, KI

Lyonsiidae

Lyonsia alvarezii Orbigny, 1846

MR

Lyonsia arcaeformis Martens, 1885 MR, FI, SG,

$\mathrm{DS}, \mathrm{AD}, \mathrm{RS}$

Lyonsia fretalis Dall, 1915

MR

Mytilimera falklandica Preston, 1913

MR, FI

Entodesma chilensis Philippi, 1845

$\mathrm{RC}, \mathrm{MR}$

Entodesma cuneata (Gray, 1828)

MR

Entodesma patagonica (Orbigny, 1846) MR

\section{Poromyidae}

Poromya adelaidis (Hedley, 1916) MR, SG, SH,

Poromya chilensis (Dall, 1908)

EL, DS, AD, RS

Poromya mactroides Dall, 1889 EC, PE, RC, MR

Poromya sp.

MR

Cuspidariidae

Cuspidaria infelix Thiele, 1912 MR, SG, SO, 
SH, PI, WS, DS, AD, OL, RS, BS, PA, BI Cuspidaria patagonica Smith, $1885 \quad$ MR Cuspidaria tenella Smith, 1912 MR, FI, BB, SR, SG, SS, SO, SH, PI, WS, EL, DS, RS, PA Cuspidaria chilensis Dahl, $1908 \quad$ RC, MR Verticordiidae

Lyonsiella radiata Dall, 1889

MR

\section{Abbreviations used for geographic areas or islands:}

AD - Adelie Land; AU - Auckland Island; BB - Burdwood Bank; BI - Bouvet Island; BS.- Bellingshausen Sea; CA - Campbell Island; CI - Crozet Island; DS - Davis Sea; EC - Ecuador; EL - Enderby Land; FI - Falkland Islands; HI - Heart Island; KI - Kerguelen Islands; MA - Macquarie Island; MB Mary Byrd Land; MI - Marion Island; MR - Magellan region; OL - Oates Land; PA - Palmer Archipelago; PE - Peru; PI - Antarctic Peninsula; RA Argentina; RC - Chile; RS - Ross Sea; SG - South Georgia; SH - South Shetland Islands; SO - South Orkney Islands; SR - Shag Rocks; SS - South Sandwich Islands; WS - Weddell Sea.

\section{DISCUSSION}

The species checklist for the Magellan molluscs summarizes the present knowledge of the distribution of the known species. Because the focus lies on the zoogeography of the species, species synonyms are not presented herein. This is the task of taxonomic contributions.

The most recent checklist for Mollusca of the Magellan region was published by Carcelles and Williamson (1951) nearly 50 years ago. It comprised 614 species, 276 families and 125 genera of the taxa Polyplacophora (45 spp./ 15 gen./ 6 fam.), Aplacophora (0/0/0), Gastropoda (352/163/67), Scaphopoda (4/1/1), Bivalvia (184/82/42) and Cephalopoda (27/15/9). They considered the region between $37^{\circ} \mathrm{S}$ on the Pacific coast (Concepción) and $43^{\circ} \mathrm{S}$ on the Atlantic side (Golfo Nuevo) and included the Falkland Islands and Burdwood Bank.

Powell (1960) published a systematic checklist for Antarctic and Subantarctic Mollusca excluding the mainland of the Magellan region but including the Falkland Islands; however, he only mentioned the species of the Magellan region in footnotes.

Recent studies on taxonomy (e.g. Ponder and Worsfold, 1994; Di Geronimo et al., 1995; Schrödl,
1996) have either increased the number of species or decreased the number due to synonymizations of species (e.g. McLean, 1984; Castellanos, 1989 1993). New zoogeographic records are the result of recent collections; e.g. Linse (1997) recorded 43 species - 8 Aplacophora, 11 Gastropoda, 2 Scaphopoda, 23 Bivalvia - new for the Magellan region and collected in sublittoral waters of the Beagle Channel. According to Dell (1971) and Brattström and Johanssen (1983), the northern boundaries of the Magellan region lie at about $41^{\circ} \mathrm{S}$, some degrees further south on the Pacific coast than defined by Carcelles and Williamson (1951). Due to the new records and these inconsistent zoogeographic definitions an updated Magellan mollusc checklist was urgently needed.

This present list comprises 397 species of Mollusca belonging to 211 genera and 101 families, excluding Polyplacophora and Cephalopoda. 10 species of Aplacophora of 7 genera and 5 families occur in the Magellan region, 250 species of Gastropoda (124 gen./ 58 fam.), 6 species of Scaphopoda (5 gen./ 2 fam.) and 131 species of Bivalvia (75 gen./ 36 fam.). Some families, like the Fissurellidae (McLean, 1984), Littorinidae and Rissoidae (Ponder and Worsfold, 1994), or Nudibranchia (Schrödl, 1999), have already been revised but revisions for others, such as the Trochidae, Pectinidae or Carditidae, are still waiting. Dr. B. Sirenko is currently revising the Polyplacophora collected during the "Victor Hensen" campaign.

\section{ACKNOWLEDGEMENTS}

I am grateful to M. Schrödl for providing his partly unpublished Nudibranchia data base. I thank Dr. A. Crame and Prof. A. Clarke for discussions about zoogeographic relationships of the Southern Ocean molluscan fauna. Thanks also to Prof. A. Brandt for helpful comments on the manuscript. The study was supported by the DFG (German Science Foundation) grant BR 1121/4-1.

\section{REFERENCES}

Bergh, L. S. R. - 1884. Report on the Nudibranchiata. Rep. Sci. Res. Challenger Zool., 10: 1-151.

Brandt, A., Linse, K. and U. Mühlenhardt-Siegel. - 1999. Biogeography of Crustacea and Mollusca of the Magellan and Antarctic regions. Sci. Mar., 63(Supl. 1): 383-389.

Brattström, H. and A. Johanssen - 1983. Ecological and regional 
zoogeography of the marine benthic fauna of Chile. Sarsia, 68 : 289-339.

Carcelles, A.R. and S.I. Williamson. - 1951. Moluscos marinos de la Provincia Magallánica. Rev. Mus. Arg. Cienc. Nat. "B. Rivadavia”, Cienc. Zool., 2 (5): 225-283.

Castellanos, Z.J.A. de. - 1988-93. Catálogo descriptivo de la malacofauna magallánica. Parts 1-12. Comisión de Investigaciones Cientificas, Provincia de Buenos Aires

Dall, W.H. - 1890. Scientific results of exploration by the U.S. Fish Commission steamer "Albatross", VII. Preliminary report on the collection of Mollusca and Brachiopoda obtained in 188788. Proc. U.S. Nat. Mus., 12: 219-362.

Dall, W.H. - 1908. The Mollusca and the Brachiopoda. Bull. Mus. Comp. Zool., 43: 205-487.

Dell, R.K. - 1964. Antarctic and sub-Antarctic Mollusca: Amphineura, Scaphopoda, and Bivalvia. Disc. Rep., 23: 93-250.

Dell, R.K. - 1971. The marine Mollusca of the Royal Society Expedition to southern Chile, 1958-59. Rec. Dom. Mus. (Wellington), 7 (17): 155-233.

Di Geronimo, I., S. Privitera and C. Valdovinos. - 1995. Fartulum magellanicum (Prosobranchia, Caecidae): A new species from the Magellan Province. Bol. Soc. Biol. Concepción, 66: 113-118.

D’Orbigny, A. - 1835-1846. Voyage dans l'Amérique Méridionale exécuté pendant les années 1826-1833 par Alcide d'Orbigny. 5. Mollusques. Libraire de la Société géologique de France, Paris.

Hain, S. - 1990. Die beschalten benthischen Mollusken (Gastropoda and Bivalvia) des Weddellmeeres, Antarktis. Ber. Polarforsch., 70: 1-181.

Harasewych, M.G., Y.I. Kantor and K. Linse. - subm. Parabuccinum, a new genus of Magellanic Buccinulid (Gastropoda: Neogastropoda), with a description of a new species. Proc. Biol. Soc. Wash.

King, P.P. and W.J. Broderip. - 1831. Description of the Cirrhipedia, Conchifera and Mollusca formed by the officers of H.M.S. Adventure and Beagle employed between the years 1826 and 1830 in surveying the southern coasts of South America including the Straits of Magelhaens and the coast of Tierra del Fuego. Zool. J., 5: 332-349.

Linse, K. - 1997. Die Verbreitung epibenthischer Mollusken im chilenischen Beagle-Kanal. Ber. Polarforsch., 228: 1-131.

Linse, K. and A. Brandt. - 1998. Distribution of epibenthic Mollusca on a transect through the Beagle Channel (southern Chile). $J$. Mar. Biol. Ass. U.K., 78: 875-889.

Mabille, J. and A.T. Rochebrune. -1889 . Mollusques. Mission scientifique du Cap Horn, 1882-3. 6, Zoologie 2: 1-143.

Marcus, E. - 1959. Lamellariacea and Opisthobranchia. Lunds Univ. Arsskr., 55 (9): 1-133.

McLean, J.H. - 1984. Systematics of Fissurella in Peruvian and Magellanic faunal provinces (Gastropoda: Prosobranchia). Contr. Sci. Nat. Hist. Mus. L.A. Cty., 354: 1-70.

Melvill, J.C. and R. Standen. - 1907. The marine Mollusca of the Scottish National Antarctic Expedition. Trans. Roy. Soc. Edinb., 46: 119-157.

Melvill, J.C. and R. Standen. - 1912. The marine Mollusca of the Scottish National Antarctic Expedition. Part II. Trans. Roy. Soc. Edinb., 46: 333-366.
Mühlenhardt-Siegel, U. - 1989. Antarktische Bivalvia der Reisen des FS "Polarstern" und des FFS "Walter Herwig" aus den Jahren 1984 bis 1986. Mitt. Hamb. Zool. Mus. Inst., 86: 153178 .

Odhner, N.H. - 1926. Die Opisthobranchien. Further Zool. Res. Swedish Antarct. Exp. 1901-1903, 2: 1-100.

Osorio, C. and N. Bahamonde. - 1970. Lista preliminar de Lamelibranquios de Chile. Bol. Mus. Nac. Hist. Nat., 31: 185-256.

Pelseneer, P. - 1903. Mollusques (Amphineures, Gastéropodes et Lamellibranches). Résultat du Voyage du S.Y. "Belgica" en 1897-1898-1899. Rappts Sci. Zool.: 1-85.

Philippi, R.A. - 1845. Diagnosen einiger neuen Conchylien. Arch. Naturgeschichte, 11: 50-71.

Ponder, W.F. and T.M. Worsfold. - 1994. A review of the rissoiform gastropods of southwestern South America (Mollusca, Gastropoda). Contr. Sci. Nat. Hist. Mus. L.A. Cty., 445: 1-63.

Powell, A.W.B. - 1951. Antarctic and sub-Antarctic Mollusca: Pelecypoda and Gastropoda. Disc. Rep., 26: 49-196.

Powell, A.W.B. - 1960. Antarctic and sub-Antarctic Mollusca. Rec. Auckl. Inst. Mus., 5 (3): 117-193.

Powell, A.W.B. - 1965. Mollusca of the Antarctic and Subantarctic Seas. In: J. van Miegham and P. van Oye (eds.), Biogeography and Ecology in Antarctica, pp. 333-380. Junk, The Hague.

Schrödl, M. - 1996. Nudibranchia y Sacoglossa de Chile: Morfología exterior y distribución. Gayana, Zool., 60 (1): 17-62.

Schrödl, M. - 1997. Range Extensions of Magellanic Nudibranchs (Ophisthobranchia) into the Peruvian Faunal Province. Veliger, 40: $38-42$

Schrödl, M. - 1999. Zoogeographic relations of Magellan Nudibranchia (Mollusca: Opisthobranchia), especially to Antarctic waters. Sci. Mar., 63(Supl. 1): 409-416.

Smith, A.E. - 1881. Mollusca and Molluscoidea. Account of the Zoological collections made during the survey of H.M.S. "Alert" in the Straits of Magellan and on the coast of Patagonia. Proc. Zool. Soc., London: 22-44.

Smith, A.E. - 1885. Report on the Lamellibranchiata collected by H.M.S. "Challenger", during the years 1873-76. Reports on the Scientific Results of the Voyage of the H.M.S. "Challenger" (1873-1876) Zoology, 13: 1 - 341.

Soot-Ryen, T. - 1959. Pelecypoda. Lunds Univ. Årsskr., 55 (6): 1-86.

Strebel, H. - 1904. Beiträge zur Kenntnis der Mollusken Fauna der Magalhaen Provinz. Zool. Jahrb. Abt. Syst., Geogr. Biol., Jena, 21: 171-248.

Strebel, H. - 1905 a. Beiträge zur Kenntnis der Mollusken Fauna der Magalhaen Provinz. II. Die Trochiden. Zool. Jahrb. Abt. Syst., Geogr. Biol., Jena, 21, Suppl. VIII: 121-166.

Strebel, H. - 1905 b. Beiträge zur Kenntnis der Mollusken Fauna der Magalhaen Provinz. 3. Zool. Jahrb. Abt. Syst., Geogr. Biol., Jena, 22: 575-666.

Strebel, H. - 1906. Beiträge zur Kenntnis der Mollusken Fauna der Magalhaen Provinz. 4. Zool. Jahrb. Abt. Syst., Geogr. Biol., Jena, 24: 91-174.

Strebel, H. - 1907. Beiträge zur Kenntnis der Mollusken Fauna der Magalhaen Provinz. 5. Zool. Jahrb. Abt. Syst., Geogr. Biol., Jena, 25: 79-196.

Strebel, H. - 1908. Die Gastropoden. Wiss. Ergebn. Schwed. Südpol.-Exped. (1901-1903), 6: 1-112. 